\title{
Preference and Practice of Traditional Medicine and Associated Factors in Jimma Town, Southwest Ethiopia
}

\author{
Belachew Umeta Chali (D), Abush Hasho, and Nimona Berhanu Koricha \\ School of Pharmacy, Institute of Health, Jimma University, Jimma, Oromia, Ethiopia \\ Correspondence should be addressed to Belachew Umeta Chali; belachewbilli2003@gmail.com
}

Received 8 March 2021; Revised 22 April 2021; Accepted 21 May 2021; Published 30 May 2021

Academic Editor: Yoshiki Mukudai

Copyright ( 2021 Belachew Umeta Chali et al. This is an open access article distributed under the Creative Commons Attribution License, which permits unrestricted use, distribution, and reproduction in any medium, provided the original work is properly cited.

\begin{abstract}
Background. Traditional medicine is the sum total of knowledge, talents, and practices that are used to uphold health, as well as to avert, identify, improve, or treat illnesses. Sociodemographic/economic characteristics, culture, and environment can influence the preference and practice of traditional medicine. Objective. To assess the preference and practices of traditional medicine and associated factors. Methods. A community-based cross-sectional study was conducted among 271 residents of Jimma town. The data were collected by interviewing selected households. The households were selected by a simple random sampling technique. The data were analysed using SPSS version 20. Descriptive statistics were used for organizing, describing, and summarizing the data. Chi-square $\left(X^{2}\right)$ test was used to identify factors associated with the preference and practice of traditional medicine. Results. More than half $(221(81.5 \%))$ of the participants practiced traditional medicine. Religion $\left(X^{2}=17.18 ; p=0.001\right)$, marital status $\left(X^{2}=15.42 ; p=0.001\right)$, occupation $\left(X^{2}=19.74 ; p=0.001\right)$, and educational level $\left(X^{2}=28.39 ; p \leq 0.001\right)$ were the sociodemographic factors determining the use of traditional medicine. However, $25(9.2 \%)$ of the participants preferred to use traditional medicine. Affordability $(25(100 \%))$, religious affiliation $(21(84 \%))$, and distance from home $(20(80 \%))$ were some reasons for preference. Educational level $\left(X^{2}=15.56 ; p=0.04\right)$, marital status $\left(X^{2}=12.39 ; p=0.04\right)$, and occupation $\left(X^{2}=15.61 ; p=0.003\right)$ were the factors affecting their preference for traditional medicine. Conclusion. A majority of the participants practiced traditional medicine use. Religion, marital status, occupation, and educational level were factors affecting the practice of the participants. More than half of the participants did not prefer to use traditional medicine. Affordability, religious affiliation, and distance from home were some reasons for preferring traditional medicine.
\end{abstract}

\section{Introduction}

Traditional medicine, an art of treatment practices, strategies, knowledge, and beliefs including plant-, animal-, and mineral-based medicines, spiritual therapies, manual techniques, and workouts applied singularly or in combination to treat, identify, and prevent illnesses or uphold the well-being. Since ancient times, humans have been using natural products, such as plants, animals, microorganisms, and oceanic organisms, in remedies to prevent or treat illnesses $[1,2]$. The recognition of these practices as standard healthcare possibilities will have an incredible influence in the cost of healthcare interventions, protective medicine, and self-healing $[3,4]$.

As to the WHO, $65-80 \%$ of the world's healthcare practice includes the use of traditional medicine in some way
[5]. There has been a continuing demand for and popular use of traditional medicine worldwide [6]. In some lower- and middle-income countries, traditional healers remain the only or main health providers for millions of people living in rural areas. For instance, the ratio of traditional health experts to the population in Africa is $1: 500$, while the ratio of physicians to the population is $1: 40,000$ [7].

In Africa, traditional medicine is part of the first set of response mechanisms for medical emergencies, whereas in others, the whole health system of the community relies on medicines embedded in indigenous practice and belief [8]. This is due to the fact that modern pharmaceuticals and medical procedures remain unreachable to a large number of African people due to their relatively high cost and concentration of health facilities in urban centers $[9,10]$. 
Despite the high consideration given to the traditional medicine practice around the world, it seems to face noteworthy challenges [3]. The most important recognized challenge is the lack of a reference standard for determining the proper dosage of the traditional medicine for the patients. This in turn has resulted in the creation of incorrect and incomplete information about the traditional medicine drugs. Another very important challenge is the lack of national policy to manage and legalize the practice of using traditional medicine [11].

In the Ethiopian context, there seems to be no village, town, or city where traditional medicine is not involved in the delivery of healthcare since it is an integral part of the local culture and accessible to the majority of the population [12]. Contrary to its wide usage, traditional medicine in Ethiopia is not uniformly practiced. The users are considerably diverse and significantly vary between regions [13]. The standard policies and regulations governing traditional medicine are too weak. Instead, the users are associated with cultural beliefs. Subsequently, traditional medicine is an integral part of a community's identity and value in that it is difficult to regulate through a nationwide framework concerning its safety and effectiveness using different medicine categorizations and descriptions $[14,15]$.

With regard to determinants of traditional medicine practice in Ethiopia, some literature studies hypothetically linked the affordability of traditional medicine to the fact of its use by up to $80 \%$ of the population, while others added deprived access to modern healthcare facilities [16-18]. Cultural belief, monthly income, and religious belief have also been mentioned as determinants $[17,19]$. Yet, there is still scarcity of information about the common preferable traditional medicine practices and reasons contributing to the link between traditional medicine practice and sociodemographic, economic, or individual difference and environmental factors.

Therefore, it is appropriate to assess the preference and practice of traditional medicine and its determinants and reasons for preferring the practices and possible factors behind the common traditional medicine being practiced among the community. So, the current study aimed to assess the preference and practice of traditional medicine and associated factors. The result of the study can be used as an input for policy makers and other stakeholders to identify and prepare directives to control and legalize the traditional medicine practices.

\section{Materials and Methods}

2.1. Study Area and Period. The study was conducted in Jimma town. The town is located at $352 \mathrm{~km}$ southwest of Addis Ababa, the capital city of Ethiopia. According to the Central Statistical Agency (CSA, 2007) report, the total population of the town was 120,960 , of whom 60,824 were males. A total of 32,191 households were present in the town [20]. According to the data from the Jimma Zone Health Bureau, there are five public health centers, one medical center, and two general hospitals. The study was conducted from November to December 2020.
2.2. Study Design. It is community-based cross-sectional study design.

2.3. Participants and Sampling Procedure. The sample size was determined using the simple population proportion formula for the cross-sectional study, $n=Z^{2} P(1-P) / d^{2}$, where $P=80 \%$ prevalence of traditional medicine use [18] and $Z=1.96$ is the value under the standard normal table for the confidence level of $95 \%$, margin of error $5 \%$, and nonresponse rate of $10 \%$. Finally, 271 households were used as a sample size, and adults older than 18 years and lived for at least 6 months were included in the study. Parents who are unable to give the required information due to any reason were excluded from the study. Priority was given for mothers and fathers, and if they are unavailable during data collection, any individual older than 18 years was included.

2.4. Data Collection Tools and Techniques. A pretested, structured questionnaire was adopted from a previously conducted study with some modifications based on the local context $[18,19]$. The pretest was conducted on $5 \%$ nonsampled respondents to check the validity, reliability, and clarity of the questionnaire. The questionnaire was prepared in English. It consists of the following parts: baseline information (age, sex, ethnicity, religion, marital status, occupation, educational level, and monthly income), the practice of traditional medicine (ever heard of traditional medicine before, current use of TM, frequency of use, behavior of users to inform their physicians, safe to mix TM with modern medicine, common types of traditional medicine used, by whom traditional medicine was practiced, and from whom information was obtained about traditional medicine use), and preference (preference between traditional and modern medicine and reason for the preference of traditional medicine). Data were collected using structured interview questionnaires. The list of all households in the town was obtained from the Jimma town Administration Office. The list was arranged in the ascending order according to the registration number given for each house by the town administration. Finally, a simple random sampling technique was used to select the required quantity of households. Trained graduating pharmacy students collected the data.

2.5. Data Processing and Analysis. The data were entered and cleaned by using Epi Info software and exported to SPSS version 20 for further analysis. Descriptive statistics were used for organizing, describing, and summarizing the data. Chi-square $\left(X^{2}\right)$ was used to check the association of independent variables with the dependent variable. $P$ value $<0.05$ was used to declare the significant association.

2.6. Ethical Consideration. The study protocol was reviewed and approved by the Ethical Review Committee of Jimma University (JU). The Ethical Review Committee of Jimma University accepted and approved verbal informed consent. 
Verbal informed consent was obtained from the participants after the purpose and methods of the study had been explained in detail, and all of their responses were kept confidential and anonymous.

\section{Results}

3.1. Sociodemographic Characteristics. A majority of the respondents were females and married. More than half of the respondents were Muslims. More than half of the participants had a monthly income of $<2000$ birr which is about 50.1 USD (Table 1).

3.2. Practice of Traditional Medicine. The prevalence of TM practice was $211(81.5 \%)$. Of those who practiced traditional medicine, $160(72.4 \%)$ of them informed their physician about the traditional medicine they used. 184 (83.3\%) of them reported that they only have symptomatic relief after the use of traditional medicine. More than half (120 $(54.3 \%))$ of the respondents obtained information on the use, benefit, and efficacy of traditional medicine from their family members. The common ailments for which traditional medicine was used were cough, headache, and intestinal/stomach infections. The majority of the participants reported that medical herbs (118 (54.4\%)) are the commonly used traditional medicine type. Most (137 $(62 \%))$ of the participants did not check the safety of traditional medicine when they used it in combination with a prescription drug, and $141(63.8 \%)$ of the participants obtained traditional medicine they used from traditional medicine healer (Table 2).

3.3. Preference of the Community for the Management of Different Ailments for the Future. The majority (204 (75.3\%)) of them preferred modern medicine, and only 25 (9.2\%) of the participants preferred the use of traditional medicine for any types of ailments. The reasons for the preference of traditional medicine were affordability $(25(100.0 \%))$ and religious affiliation (21 (84.0\%)) (Table 3).

3.4. Factors Affecting the Practice and Preference of Traditional Medicine. Religion, marital status, occupation, and educational level were the factors affecting the practice of traditional medicine in the study area. Marital status, occupation, and educational level were the sociodemographic characteristics affecting their preference (Table 4).

\section{Discussion}

In this study, the practice of traditional medicine was $81.5 \%$. The finding was higher than the study conducted in North Central Ethiopia [21] which states that the practice of traditional medicine use is $35.8 \%$. However, the practice is lower than the study conducted in Burka Jato Kebele, West Ethiopia [22], in which $92.22 \%$ of the participants rely on traditional medicine. The discrepancy might be due to the sample size difference and accessibility of health facilities. And it is almost comparable with the study conducted in Shoba Bultum, Southwest Ethiopia [19], in which $79.47 \%$ of the participants practice traditional medicine use. From those participants who used traditional medicines, $72.4 \%$ of the participants reported to their physicians that they used traditional medicine, and $83.4 \%$ of them felt that traditional medicine use for the management of different ailments only gives symptomatic relief.

Around fifty-four percent of the participants had information about the use of traditional medicine for different ailments from their family, and the most common traditional medicine used was medical herbs (53.4\%), and the finding was similar to the study conducted in North Central, West, and Southeast Ethiopia [21-23]. The most common ailments for which the community uses those traditional medicines were cold/influenza/cough, headache, and intestinal infections.

In this study, $75.3 \%$ of the participants prefer to use modern medicine for any type of ailments. The report value was smaller than the study conducted in North Central and Southeast Ethiopia $[21,23]$ in which $69.7 \%$ and $75.52 \%$ of the participants prefer to use traditional medicines. The dissimilarity might be due to the social, cultural, and economic difference of study areas.

Different factors affected the practice of traditional medicine in the study area, from which religion, marital status, and occupation were the factors determining the practice of traditional medicine in the study area. This might be due to community perception for some diseases to be cured by traditional medicine only [22]. The other factors affecting the practice of traditional medicine were marital status and occupation. The cost to use traditional and modern medicines is different and cheap for traditional medicine use which might be the reason for the association. The report was comparable with the study conducted in Northwest Ethiopia which indicated the relationships of occupations with knowledge, attitude, and practice of traditional medicine [18] and the study conducted in Burka Jato Kebele which reported the significance relationship between the income and practice of traditional medicine [22].

Only $9.2 \%$ of the participants preferred to use traditional medicine. The figure was different from previous studies $[22,23]$ which reported that $31.85 \%$ and $28.8 \%$ of the participants preferred to use traditional medicine. This difference might be due to the difference in the selection of the study site as the current study was conducted in the urban population. According to the present study, religious affiliation, therapeutic effectiveness, affordability, distance from home, and culture were reasons of the studied community to prefer traditional medicine. The finding of the study was comparable with the study conducted in Burka Jato Kebele [22]. The study conducted in Wayu, Western Ethiopia, also reported that the effectiveness of traditional medicine was the reason for preference [24]. The study also reported that there is a significant relationship between the marital status and the preference of the participants for the healthcare service in the future. 
TABLE 1: Sociodemographic characteristics of study participants, Jimma town, 2020.

\begin{tabular}{|c|c|c|c|}
\hline Sociodemographic characteristics & Classification & Frequency & Percentage (\%) \\
\hline \multirow{5}{*}{ Age } & $18-27$ & 95 & 35.1 \\
\hline & $28-37$ & 82 & 30.3 \\
\hline & $38-47$ & 37 & 13.7 \\
\hline & $48-57$ & 33 & 12.2 \\
\hline & $>57$ & 24 & 8.9 \\
\hline \multirow{2}{*}{$\operatorname{Sex}$} & Male & 120 & 44.3 \\
\hline & Female & 151 & 55.7 \\
\hline \multirow{5}{*}{ Ethnicity } & Oromo & 218 & 80.4 \\
\hline & Amhara & 31 & 11.4 \\
\hline & Gurage & 6 & 2.2 \\
\hline & Tigre & 2 & .7 \\
\hline & Others $^{\mathrm{a}}$ & 14 & 5.2 \\
\hline \multirow{4}{*}{ Religion } & Muslim & 155 & 57.2 \\
\hline & Orthodox & 66 & 24.4 \\
\hline & Protestant & 47 & 17.3 \\
\hline & Others $^{\mathrm{b}}$ & 3 & 1.1 \\
\hline \multirow{4}{*}{ Marital status } & Single & 62 & 22.9 \\
\hline & Married & 197 & 72.7 \\
\hline & Divorced & 9 & 3.3 \\
\hline & Widowed & 3 & 1.1 \\
\hline \multirow{7}{*}{ Occupation } & Farmer & 53 & 19.6 \\
\hline & Government worker & 37 & 13.7 \\
\hline & Housewife/husband & 57 & 21.0 \\
\hline & Private business owner & 51 & 18.8 \\
\hline & Student & 32 & 11.8 \\
\hline & Daily worker & 21 & 7.7 \\
\hline & Others $^{c}$ & 20 & 7.4 \\
\hline \multirow{5}{*}{ Educational level } & Not read and write & 58 & 21.4 \\
\hline & $7-12$ & 74 & 27.3 \\
\hline & Read and write & 62 & 22.9 \\
\hline & $>12$ & 62 & 22.9 \\
\hline & $1-6$ & 15 & 5.5 \\
\hline \multirow{5}{*}{ Monthly family income } & $<2000$ & 106 & 39.1 \\
\hline & $2001-3800$ & 55 & 20.3 \\
\hline & $3801-6000$ & 70 & 25.8 \\
\hline & $6001-10,000$ & 31 & 11.4 \\
\hline & $>10000$ & 9 & 3.3 \\
\hline
\end{tabular}

${ }^{a}$ Silte, Dawro, and Yem. ${ }^{b}$ Waaqeffannaa and catholic. ${ }^{\mathrm{c}}$ Carpenter and driver.

Table 2: Practice of traditional medicine in Jimma town, Southwest Ethiopia, 2020.

Have you heard of traditional medicine? $(n=271)$

Frequency

Percentage (\%)

Yes

269

99.3

No

2

0.7

Traditional medicine practice $(n=271)$

Yes

221

81.5

No

50

Types of ailments

Cold/influenza/cough

133

60.2

Headache

110

49.8

Stomach/intestinal infections

86

38.9

Others $^{\circledR}$

$34 \quad 15.4$

How frequently have you used traditional medicine? $(n=221)$

Daily 
TABle 2: Continued.

Have you heard of traditional medicine? $(n=271)$

\begin{tabular}{|c|c|c|}
\hline & Frequency & Percentage $(\%)$ \\
\hline Sometimes & 124 & 56.1 \\
\hline When unwell & 91 & 41.2 \\
\hline \multicolumn{3}{|c|}{ Behavior of users of traditional medicine to inform their physicians $(n=221)$} \\
\hline Yes & 160 & 72.4 \\
\hline No & 61 & 27.6 \\
\hline \multicolumn{3}{|c|}{ If you took traditional medicine whilst on prescription medicine, did you check it was safe to mix? $(n=221)$} \\
\hline Yes & 84 & 38.0 \\
\hline No & 137 & 62.0 \\
\hline \multicolumn{3}{|c|}{ Outcome of using traditional medicine for the management of ailments $(n=221)$} \\
\hline Symptomatic relief & 184 & 83.3 \\
\hline Permanent cure & 35 & 15.8 \\
\hline No change & 2 & 0.9 \\
\hline \multicolumn{3}{|c|}{ Common types of traditional medicines used $(n=221)$} \\
\hline Medical herbs & 118 & 53.4 \\
\hline Faith healer/holy water & 91 & 41.2 \\
\hline Bone setter & 5 & 2.3 \\
\hline Medical herbs and holy water & 3 & 1.4 \\
\hline Medical herbs, holy water, and bone setter & 4 & 1.8 \\
\hline \multicolumn{3}{|c|}{ Sources of information about traditional medicine use $(n=221)$} \\
\hline Family & 120 & 54.3 \\
\hline Friends & 96 & 43.4 \\
\hline Healthcare practitioners & 5 & 2.3 \\
\hline \multicolumn{3}{|c|}{ By whom traditional medicine was practiced? $(n=221)$} \\
\hline Traditional medicine healer & 141 & 63.8 \\
\hline Elderly & 10 & 4.5 \\
\hline Mother and father & 70 & 31.7 \\
\hline
\end{tabular}

${ }^{\circledR}$ Fever, hypertension, and arteritis/joint pain.

TABLE 3: Preference of the community for traditional medicine and reason for preference, Jimma town, Southwest Ethiopia, 2020 ( $N=271)$.

\begin{tabular}{lcc}
\hline Preference & Frequency & Percentage $(\%)$ \\
\hline Traditional medicine & 25 & 9.2 \\
Modern medicine & 204 & 75.3 \\
Both & 42 & 15.5 \\
\hline Reason for the preference of traditional medicine $(N=25)^{*}$ & & \\
Religious affiliations & 21 & 84.0 \\
Therapeutic effectiveness & 20 & 80.0 \\
Affordability & 25 & 100.0 \\
Distance from home & 20 & 80.0 \\
Cultural influence & 14 & 56.0 \\
\hline
\end{tabular}

${ }^{*}$ The respondents respond more than one response, so the percentage might be greater than $100 \%$.

TABLE 4: Relationships of the sociodemographic distribution of participants with the preference and practice of traditional medicine in Jimma town, Southwest Ethiopia, 2020.

\begin{tabular}{|c|c|c|c|c|c|c|c|c|c|}
\hline \multirow{2}{*}{ Variables } & \multicolumn{2}{|c|}{ Practice } & \multirow{2}{*}{ Test statistics } & \multirow{2}{*}{$p$ value } & \multicolumn{3}{|c|}{ Preference } & \multirow{2}{*}{ Test statistics } & \multirow{2}{*}{$p$ value } \\
\hline & Yes & No & & & TM & MM & Both & & \\
\hline \multicolumn{10}{|l|}{ Sex } \\
\hline Male & 100 & 20 & \multirow{2}{*}{$X^{2}=0.65$} & \multirow{2}{*}{0.42} & 7 & 97 & 16 & \multirow{2}{*}{$X^{2}=4.22$} & \multirow{2}{*}{0.12} \\
\hline Female & 120 & 31 & & & 18 & 107 & 26 & & \\
\hline \multicolumn{10}{|l|}{ Ethnicity } \\
\hline Oromo & 182 & 36 & \multirow{5}{*}{$X^{2}=6.62$} & \multirow{5}{*}{0.16} & 23 & 157 & 38 & \multirow{5}{*}{$X^{2}=10.38$} & \multirow{5}{*}{0.24} \\
\hline Amhara & 24 & 7 & & & 1 & 27 & 3 & & \\
\hline Tigre & 1 & 1 & & & 0 & 1 & 1 & & \\
\hline Gurage & 6 & 2 & & & 0 & 6 & 0 & & \\
\hline Others $^{\mathrm{a}}$ & 7 & 5 & & & 1 & 13 & 0 & & \\
\hline
\end{tabular}


TABLE 4: Continued.

\begin{tabular}{|c|c|c|c|c|c|c|c|c|c|}
\hline \multirow{2}{*}{ Variables } & \multicolumn{2}{|c|}{ Practice } & \multirow{2}{*}{ Test statistics } & \multirow{2}{*}{$p$ value } & \multicolumn{3}{|c|}{ Preference } & \multirow{2}{*}{ Test statistics } & \multirow{2}{*}{$p$ value } \\
\hline & Yes & No & & & TM & MM & Both & & \\
\hline \multicolumn{10}{|l|}{ Religion } \\
\hline Muslims & 138 & 17 & \multirow{4}{*}{$X^{2}=15.88$} & \multirow{4}{*}{0.001} & 17 & 110 & 28 & \multirow{4}{*}{$X^{2}=6.52$} & \multirow{4}{*}{0.37} \\
\hline Protestants & 31 & 16 & & & 3 & 39 & 5 & & \\
\hline Orthodox & 49 & 17 & & & 4 & 59 & 9 & & \\
\hline Others $^{\mathrm{b}}$ & 2 & 1 & & & 1 & 1 & 0 & & \\
\hline \multicolumn{10}{|l|}{ Marital status } \\
\hline Married & 171 & 26 & \multirow{4}{*}{$X^{2}=14.92$} & \multirow{4}{*}{0.002} & 21 & 139 & 37 & \multirow{4}{*}{$X^{2}=12.39$} & \multirow{4}{*}{0.05} \\
\hline Single & 41 & 21 & & & 3 & 54 & 5 & & \\
\hline Divorced & 6 & 3 & & & 0 & 9 & 0 & & \\
\hline Widowed & 2 & 1 & & & 1 & 2 & 0 & & \\
\hline \multicolumn{10}{|l|}{ Occupation } \\
\hline Farmer & 50 & 3 & \multirow{5}{*}{$X^{2}=19.95$} & \multirow{5}{*}{0.001} & 10 & 32 & 11 & \multirow{5}{*}{$X^{2}=15.61$} & \multirow{5}{*}{0.05} \\
\hline Government employee & 25 & 12 & & & 1 & 32 & 4 & & \\
\hline Housewife/husband & 53 & 4 & & & 8 & 40 & 9 & & \\
\hline Private workers & 39 & 12 & & & 2 & 42 & 7 & & \\
\hline Others $^{c}$ & 53 & 20 & & & 4 & 58 & 4 & & \\
\hline \multicolumn{10}{|l|}{ Educational level } \\
\hline Not read and write & 53 & 5 & \multirow{5}{*}{$X^{2}=26.54$} & \multirow{5}{*}{$<0.001$} & 9 & 34 & 5 & \multirow{5}{*}{$X^{2}=15.56$} & \multirow{5}{*}{0.04} \\
\hline Read and write & 55 & 7 & & & 8 & 46 & 8 & & \\
\hline $1-6$ & 14 & 1 & & & 1 & 12 & 2 & & \\
\hline $7-12$ & 61 & 13 & & & 5 & 58 & 11 & & \\
\hline$>12$ & 37 & 25 & & & 2 & 54 & 6 & & \\
\hline
\end{tabular}

${ }^{\mathrm{a}}$ Silte, Dawro, and Yem. ${ }^{\mathrm{b}}$ Waaqeffannaa and catholic. ${ }^{\mathrm{c}}$ Carpenter and driver.

\section{Conclusion}

The majority of the participants practiced traditional medicine use. More than half of the participants did not prefer to use traditional medicine. Affordability, religious affiliation, distance from home, therapeutic effectiveness, and cultural influence were reasons for preferring traditional medicine. Marital status, occupation, religion, and educational level were determinants of the preference and practice of traditional medicine.

\section{Limitations of the Study}

Relatively small sample size, being cross-sectional in design, single site, and inclusion of only urban population were the main limitations of the study.

\section{Data Availability}

The data used to support the findings of this study are included within the article.

\section{Conflicts of Interest}

The authors declare that they have no conflicts of interest.

\section{Authors' Contributions}

AH proposed and designed the study. BU implemented the study. BU and NB performed the statistical analysis and read, critically commented, and prepared the final manuscript. All authors read and approved the final manuscript.

\section{Acknowledgments}

The authors would like to acknowledge Jimma University, Jimma town Administration Office, data collectors, and participants for their unreserved assistance. The current work was funded by Jimma University.

\section{References}

[1] C. Zollman and A. Vickers, "What is complementary medicine?," British Medical Journal, vol. 319, no. 7211, pp. 693-696, 1999.

[2] WHO, Traditional Medicine Executive Board $134^{\text {th }}$ Session Provisional Agenda Item 9.1, pp. 1-4, World Health Organisation, Geneva, Switzerland, 2013.

[3] R. Abbott, Documenting Traditional Medical Knowledge, p. 48, World Intellectual Property Organisation, Geneva, Switzerland, 2014.

[4] M. M. Iwu and E. Gbodossou, "The role of traditional medicine," The Lancet, vol. 356, no. 9248, p. s3, 2000.

[5] S. Y. Pan, G. Litscher, K. Chan, Z. L. Yu, H. Q. Chen, and K. M. Ko, "Traditional medicines in the world: where to go next?" Evidence-based Complementary and Alternative Medicine, vol. 2014, Article ID 739895, 4 pages, 2014.

[6] World Health Organization, WHO Global report on traditional and complementary medicine 2019, pp. 1-228, World Health Organization, Geneva, Switzerland, 2019.

[7] N. L. Vecchiato, "Traditional medicine," The Ecology of Health and Disease in Ethiopia, vol. 2013, pp. 157-178, 2019. 
[8] A. A. Abdullahi, "Trends and challenges of traditional medicine in Africa," African Journal of Traditional, Complementary and Alternative Medicines, vol. 8, no. 5, pp. 115123, 2011.

[9] S. S. Antwi-Baffour, A. I. Bello, D. N. Adjei, S. A. Mahmood, and P. F. Ayeh-kumi, "The place of traditional medicine in the african society: the science, acceptance and support," American Journal of Health Research, vol. 2, no. 2, p. 49, 2014.

[10] M. E. Mothibe and M. Sibanda, "African traditional medicine: South African perspective," Traditional and Complementary Medicine, pp. 1-27, Intechopen, London, UK, 2019.

[11] R. Mirzaeian, F. Sadoughi, S. Tahmasebian, and M. Mojahedi, "Progresses and challenges in the traditional medicine information system: a systematic review," Journal of Pharmacy \& Pharmacognosy Research, vol. 7, no. 4, pp. 246-259, 2019.

[12] G. Bekele and P. R. Reddy, "Ethnobotanical study of medicinal plants used to treat human ailments by Guji Oromo tribes in Abaya District, Borana, Oromia, Ethiopia," Universal Journal of Plant Science, vol. 3, no. 1, pp. 1-8, 2015.

[13] A. B. Muluye and M. W. Ayicheh, "Medicinal plants utilized for hepatic disorders in Ethiopian traditional medical practices: a review," Clinical Phytoscience, vol. 6, no. 1, 2020.

[14] D. Adams, S. Dagenais, T. Clifford et al., "Complementary and alternative medicine use by pediatric specialty outpatients," Pediatrics, vol. 131, no. 2, pp. 225-232, 2013.

[15] World Health Organization (WHO), WHO traditional medicine strategy 2014-2023, pp. 1-76, World Health Organisation, 2013.

[16] K. Kassaye, A. Amberbir, B. Getachew, and Y. Mussema, "A historical overview of traditional medicine practices and policy in Ethiopia," Ethiopian Journal of Health Development, vol. 20, no. 2, 2007.

[17] F. Hailu, A. Cherie, T. Gebreyohannis, and R. Hailu, "Determinants of traditional medicine utilization for children: a parental level study in tole district, Oromia, Ethiopia," BMC Complementary Medicine and Therapies, vol. 20, no. 1, pp. 1-12, 2020.

[18] S. M. Wassie, L. L. Aragie, B. W. Taye, and L. B. Mekonnen, "Knowledge, attitude, and utilization of traditional medicine among the communities of Merawi town, Northwest Ethiopia: a cross-sectional study," Evidence-Based Complementary and Alternative Medicine, vol. 2015, Article ID 138073, 7 pages, 2015.

[19] T. Laelago, T. Yohannes, and F. Lemango, "Prevalence of herbal medicine use and associated factors among pregnant women attending antenatal care at public health facilities in Hossana Town, Southern Ethiopia: facility based cross sectional study," Archives of Public Health, vol. 74, no. 1, pp. 1-8, 2016.

[20] Central Statistical Authority of Ethiopia (CSA), Jimma Zone Population Survey, Central Statistical Authority (CSA) of Ethiopia, Addis Ababa, Ethiopia, 2007.

[21] T. J. Aragaw, D. T. Afework, and K. A. Getahun, "Assessment of knowledge, attitude, and utilization of traditional medicine among the communities of Debre Tabor Town, Amhara Regional State, North Central Ethiopia: a cross-sectional study," Evidence-Based Complementary and Alternative Medicine, vol. 2020, Article ID 6565131, 10 pages, 2020.

[22] A. Gari, R. Yarlagadda, and M. Wolde-Mariam, "Knowledge, attitude, practice, and management of traditional medicine among people of Burka Jato Kebele, West Ethiopia," Journal of Pharmacy and Bioallied Sciences, vol. 7, no. 2, pp. 136-144, 2015.
[23] G. Misha, R. Yarlagadda, and M. Wolde-Mariam, "Knowledge, attitude, practice and management of traditional medicine among people of Shopa Bultum, Southeast Ethiopia," Research Journal of Pharmaceutical, Biological and Chemical Sciences, vol. 5, no. 5, pp. 152-170, 2014.

[24] N. Belachew, T. Tadesse, and A. A. Gube, "Knowledge, attitude, and practice of complementary and alternative medicine among residents of wayu town, western Ethiopia," Journal of Evidence-Based Complementary and Alternative Medicine, vol. 22, no. 4, pp. 929-935, 2017. 